\title{
Simulación en tiempos de pandemia
}

\author{
Yony Fernando Ceballos ${ }^{1}$ \\ ${ }^{1}$ Universidad de Antioquia, Medellín, Colombia \\ yony.ceballos@udea.edu.co
}

(Publicado en Internet: 1 junio 2021)

La computación en América Latina y principalmente en Colombia ha tenido avances significativos en los últimos años dados por la velocidad de los cambios en esta área del conocimiento que son debidos generalmente a la aparición de nuevas tecnologías. En este sentido, se ha identificado que la aplicación de herramientas informáticas en diversos entornos de trabajo e innovación gracias al crecimiento de Internet, así como los efectos de la pandemia, están orientadas a mejorar la calidad de vida de las personas, y en general de instituciones en las cuales la forma de trabajo se ha redefinido.

Los retos en esta situación se observan en mejoras de conectividad, actividades basadas en metas, y la posible adaptación de los empleados. Es por esto qué técnicas como la simulación para la evaluación de rendimientos de redes distribuidas para quienes comparten acceso a los mismos recursos al interior de una empresa se vuelven relevantes. Estos estudios hacen necesario incluir en la fase de planeación, alguna técnica de simulación para que el decisor pueda anticiparse apropiadamente a eventos adversos, evaluación de capacidad, y evaluación de diferentes decisiones en el tiempo.

Sin embargo, es necesario que se analice cual es el tipo de problema a evaluar. Las técnicas tradicionales de simulación se agrupan en tres tipos a saber: simulación de eventos discretos, la cual permite representar procesos de decisión a nivel operativo, simulación continua que está orientada a problemas de alto nivel de agregación, y simulación basada en agentes la cual permite un modelado a diferentes niveles de abstracción, dado por la representatividad de los agentes.

La simulación discreta en los años recientes se ha centrado en evaluar la forma en la cual la distribución de personas en los entornos relacionados a sistemas de atención se distribuyen, para asi lograr mejorar los servicios, de una forma eficiente y siguiendo los protocolos de seguridad dictados por diferentes entes gubernamentales para salvaguardar la vida (Bertoglio et al., 2020; Das, 2020; Kierzkowski \& Kisiel, 2020; Lim et al., 2020). La velocidad en la cual se pueden hacer evaluaciones reales, que incluyan la distribución física, tiempo de desplazamiento, tiempos de atención y demás elementos asociados al servicio (clientes preferenciales y/o urgentes) hacen esta herramienta ideal para evaluar las políticas de distribución, distanciamiento, y demás requisitos que el mismo servicio requiera. El requerimiento de información puede variar, ya que generalmente se trabaja con variables de naturaleza estocástica y aun así, las decisiones pueden ser determinísticas.

En cuanto a simulación continua, es interesante la forma en la cual se aplica, dado que se orienta a situaciones de propagación del virus, la forma en la cual se deben hacer los procesos de cierres y evaluar la efectividad de los mismos con la creación de políticas apropiadas para tal fin (Cuadros et al., 2021; Fair et al., 2021; Ibarra-Vega, 2020; Kontogiannis, 2021). La gran ventaja de esta técnica se centra en las posibilidades de representar las poblaciones afectadas como un conglomerado, de la forma en que los epidemiólogos tradicionalmente subdividen la población según la etapa de contagio, y simplemente empleando un modelo de ecuaciones diferenciales acoplado.

Si por el contrario se desea modelar la forma en la cual cada persona como individuo heterogéneo reacciona en situaciones de pandemia, la técnica sugerida puede ser simulación basada en agentes (Araya, 2021; Cuevas, 2020; Silva et al., 2020; Tatapudi et al., 2020). Si las poblaciones no son "demasiado grandes", se puede emplear esta aproximación, en la cual cada (individuo) agente, es configurado con una estructura de toma decisiones basado en su habilidad de interactuar y responder a estímulos del entorno, configurando un patrón individual y permitiendo analizar comportamientos emergentes.

Todas estas herramientas cuentan con las ventajas para los tomadores de decisiones, ya que no involucran el contacto directo con las personas, y la información para su construcción puede obtenerse mediante encuestas, entrevistas y datos históricos que tradicionalmente se han recogido en todos los espacios donde se ofrecen servicios, configurando una relación directa con la analítica de datos y su posterior aplicación en la construcción de escenarios de prueba. 
En conclusión, es importante emplear diversas técnicas para analizar situaciones de pandemia, lograr incluir el tiempo como variable y anticiparse a situaciones que para los gobiernos suelen ser complejas, y que para las empresas pueden acarrear consecuencias nefastas.

\section{ORCID iD}

\section{Yony Fernando Ceballos iD https://orcid.org/0000-0001-5787-8832}

\section{Referencias}

Araya, F. (2021). Modeling the spread of COVID-19 on construction workers: An agent-based approach. Safety Science, 133, 105022. https://doi.org/10.1016/j.ssci.2020.105022

Bertoglio, N., Lamperti, G., Zanella, M., \& Zhao, X. (2020). Temporal-Fault Diagnosis for Critical-Decision Making in Discrete-Event Systems. Procedia Computer Science, 176, 521-530. https://doi.org/10.1016/j.procs.2020.08.054

Cuadros, D. F., Branscum, A. J., Mukandavire, Z., Miller, F. D., \& MacKinnon, N. (2021). Dynamics of the COVID19 epidemic in urban and rural areas in the United States. Annals of Epidemiology, 59, 16-20. https://doi.org/10.1016/j.annepidem.2021.04.007

Cuevas, E. (2020). An agent-based model to evaluate the COVID-19 transmission risks in facilities. Computers in Biology and Medicine, 121,103827. https://doi.org/10.1016/j.compbiomed.2020.103827

Das, A. (2020). Impact of the COVID-19 pandemic on the workflow of an ambulatory endoscopy center: an assessment by discrete event simulation. Gastrointestinal Endoscopy, 92(4), 914-924. https://doi.org/10.1016/j.gie.2020.06.008

Fair, J. M., LeClaire, R. J., Dauelsberg, L. R., Ewers, M., Pasqualini, D., Cleland, T., \& Rosenberger, W. (2021). Systems dynamics and the uncertainties of diagnostics, testing and contact tracing for COVID-19. Methods. https://doi.org/10.1016/j.ymeth.2021.03.008

Ibarra-Vega, D. (2020). Lockdown, one, two, none, or smart. Modeling containing covid-19 infection. A conceptual model. Science of The Total Environment, 730, 138917. https://doi.org/10.1016/j.scitotenv.2020.138917

Kierzkowski, A., \& Kisiel, T. (2020). Simulation model of security control lane operation in the state of the COVID19 epidemic. Journal of Air Transport Management, 88, 101868. https://doi.org/10.1016/j.jairtraman.2020.101868

Kontogiannis, T. (2021). A qualitative model of patterns of resilience and vulnerability in responding to a pandemic outbreak with system dynamics. Safety Science, 134, 105077. https://doi.org/10.1016/j.ssci.2020.105077

Lim, C. Y., Bohn, M. K., Lippi, G., Ferrari, M., Loh, T. P., Yuen, K.-Y., Adeli, K., \& Horvath, A. R. (2020). Staff rostering, split team arrangement, social distancing (physical distancing) and use of personal protective equipment to minimize risk of workplace transmission during the COVID-19 pandemic: A simulation study. Clinical Biochemistry, 86, 15-22. https://doi.org/10.1016/j.clinbiochem.2020.09.003

Silva, P. C. L., Batista, P. V . C., Lima, H. S., Alves, M. A., Guimarães, F. G., \& Silva, R. C. P. (2020). COVID-ABS: An agent-based model of COVID-19 epidemic to simulate health and economic effects of social distancing interventions. Chaos, Solitons \& Fractals, 139, 110088. https://doi.org/10.1016/j.chaos.2020.110088

Tatapudi, H., Das, R., \& Das, T. K. (2020). Impact assessment of full and partial stay-at-home orders, face mask usage, and contact tracing: An agent-based simulation study of COVID-19 for an urban region. Global Epidemiology, 2, 100036. https://doi.org/10.1016/j.gloepi.2020.100036 